\title{
The Design of Intelligent Wheelchair Based on MSP430
}

\author{
Peifen $\mathrm{Jin}^{1, \mathrm{a}^{*}}$, Yujie Chen ${ }^{1, \mathrm{~b}}$, Peixue Liu ${ }^{1, \mathrm{c}}$ \\ ${ }^{1}$ Department of Mechanical and electrical engineering ,QingDao HuangHai College, Qingdao, \\ 266427, China \\ ajinpeifen@163.com, b79572611@qq.com, cytulpx@163.com
}

Keywords: Intelligent wheelchair, PWM speed regulation, Sensor

\begin{abstract}
With the continuous development of the information society, R \& D new intelligent wheelchair products, not only can better meet the needs of the elderly, but also can help the elders to improve the degree of freedom of action, to enable them better integrated into society. This design takes the MSP430 single chip microcomputer as the core control unit, through processing the data collected by the sensor to adjust the movement state of the intelligent wheelchair.
\end{abstract}

\section{Introduction}

With the deepening of the degree of aging society, elderly care become focus of social attention, improve the convenience of the elderly action intelligent elderly care product demand is growing ${ }^{[1-2]}$.

As an important means of transport of wheelchair disabled elderly people's daily life, plays an increasingly important role ${ }^{[3]}$. However, the current electric wheelchair products only to replace the power of human power, the elderly in the use of the process is very difficult ${ }^{[4]}$. With the continuous development of the information society, R \& D new intelligent wheelchair products, not only can better meet the needs of the elderly, also can help older people to improve the degree of freedom of action, to enable them to better integrate into society $^{[5]}$. This design takes the MSP430 single chip microcomputer as the core control unit, through processing the data collected by the sensor to adjust the movement state of the intelligent wheelchair.

\section{Scheme Design}

Overall design overview

This design uses the MSP430 single chip microcomputer as the core of each module control; ultrasonic sensor and infrared sensor is used for detecting wheelchair operating situation of, smoke sensor mq-2 detection of harmful gases, and real-time transmits the signal to the microcontroller; power module with $12 \mathrm{~V}$ electric car powered by lithium battery, voltage regulator module output need $5 \mathrm{~V}, 3 \mathrm{~V}$ voltage. 


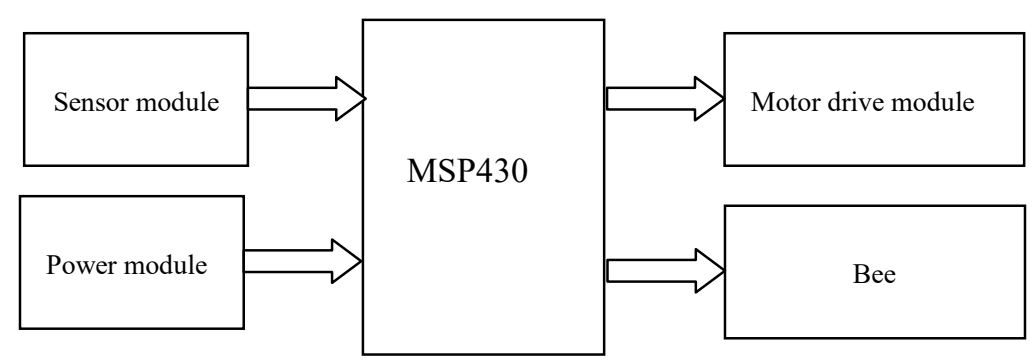

Fig.1 Overall design overview

\section{Control system}

Research focus of this design is to achieve the intelligent wheelchair's safe driving and intelligent obstacle avoidance, using multiple sensors to collect wheelchair around traffic information, the main control system need to have sufficient I / O pins, to accept both multiple sensor signals, the selection of MSP430g2553 device can meet the requirements.

\section{Sensor module}

This design uses three infrared module, two placed on the smart wheelchair central front, a placed on the smart wheelchair rear centre and on the front part of the intelligent wheelchair installation a ultrasonic module, with the ultrasonic module through the infrared module at the same time with the obstacle avoidance, which can comprehensively detect the presence of obstacles can also monitor whether there is danger of falling, the most comprehensive safe obstacle avoidance design reach the.

Motor drive module

$\mathrm{H}$ based on the PWM bridge circuit speed, can achieve continuously variable speed, the speed change is smooth, the speed regulation span is big, the overload ability is strong, can reliably complete the motor repeatedly start, brake and reverse and so on. Output control DC motor using power transistor as power amplifier. The design for the three intelligent wheelchair, two front wheels were by a DC motor drive control, by controlling the DC motor to two wheeled differential to complete steering, rear wheel Vientiane, the supporting role. The infrared module were placed in the intelligent wheelchair before and after the central, when the front part of the intelligent wheelchair cliff, the main control system will control wheel turn right, ultrasonic module is placed on the intelligent wheelchair front tilt steering gear for obstacle detection for intelligent wheelchair on both sides of the front and, when an obstacle is detected, the intelligent wheelchair will turn right, around obstacles.

\section{Power module}

Because there are lots of hardware used in this design is the need to achieve the function, so greater consumption in order to solve this problem and does not affect the performance, the design by $12 \mathrm{~V}$ electric cars powered by lithium batteries, and the voltage regulator module step-down can greatly reduce the space occupied, was able to lose, very high utilization rate of batteries. 


\section{System hardware circuit design}

Sensor module

The sensor module mainly uses the infrared sensor, the ultrasonic sensor and the gas sensor. The principle diagram of each sensor is shown in figure 2-4.
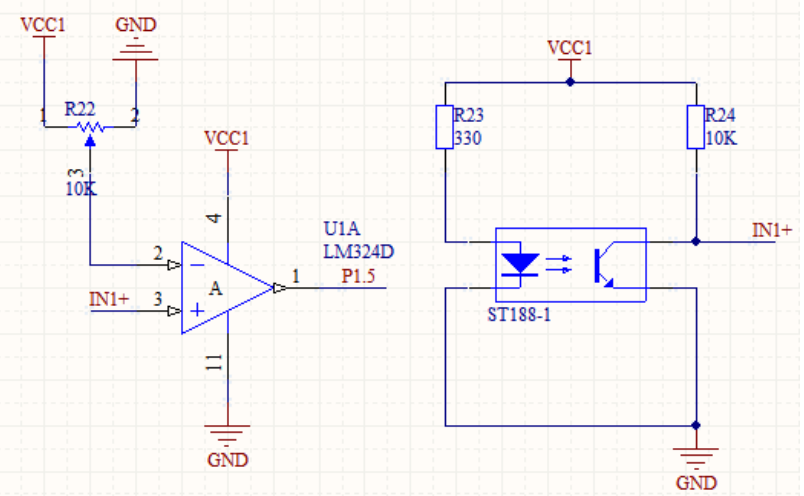

Fig.2 Infrared module circuit
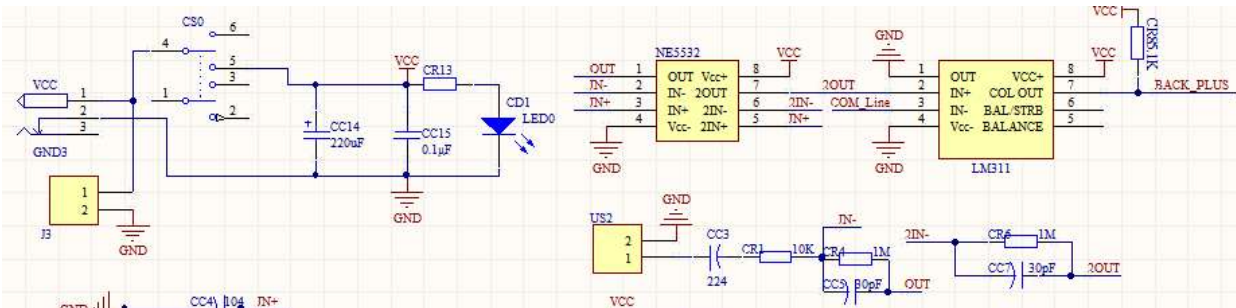

$$
\text { GND N|| }
$$

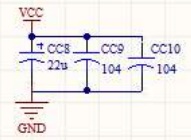

$\overbrace{1 \mathrm{M}}^{\text {CR7 }}$ BACK PIUS
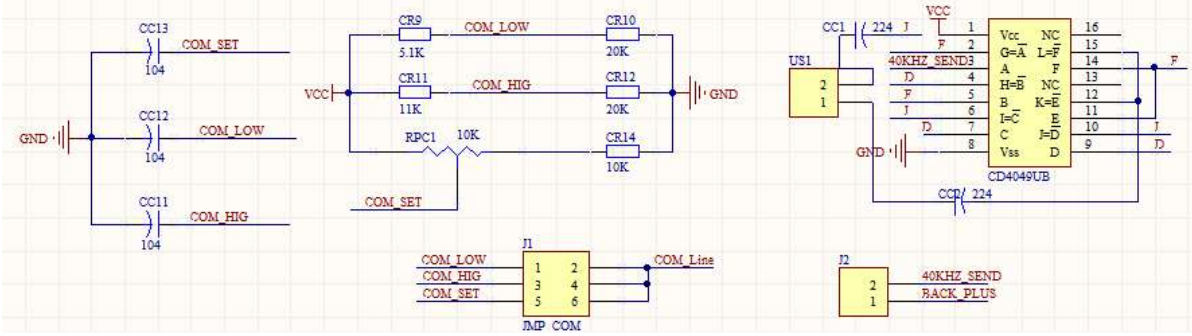

Fig.3Ultrasonic module circuit

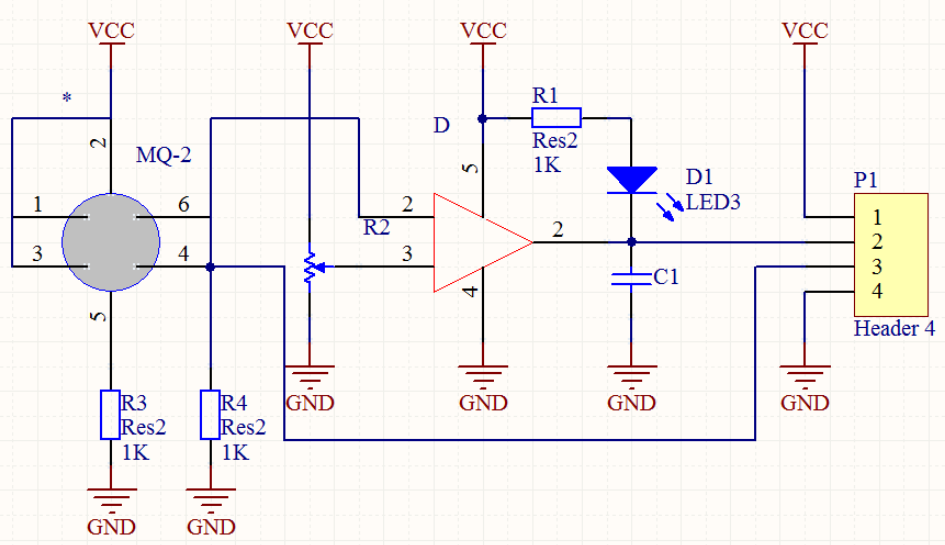

Fig.4 MQ-2 module circuit 


\section{Driving circuit}

Motor drive using $\mathrm{H}$ bridge drive circuit, through the microcontroller to give the driver circuit PWM to control the motor of the intelligent wheelchair is reversing and start and stop. Drive schematic figure5.

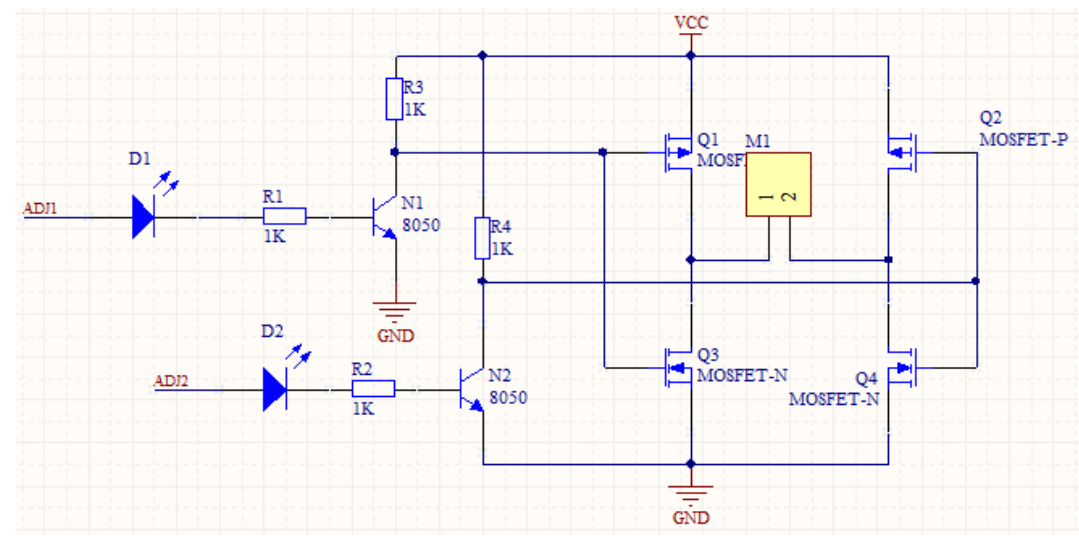

Fig.5 H bridge drive circuit

System power supply circuit

Each module of the requirements of the voltage is different, the need for a single chip voltage of $3 \mathrm{~V}$, and the voltage required for the motor is $5 \mathrm{~V}$. This design is used in the $12 \mathrm{~V}$ lithium battery power supply, through the $5 \mathrm{~V}$ to get the LM7805 voltage, through the LM7111 to get the $3.3 \mathrm{~V}$ voltage.

Main control circuit

We use the PWM (Pulse Width Modulation) to carry on the electric motor's speed control, the PWM signal is produced by the single chip microcomputer software, uses the PWM method to be able to realize the speed regulation very easily.

\section{Software design}

This design uses the MSP430 microcontroller, select IAR as the programming software. A total of $16 \mathrm{I} / \mathrm{O}$ port using p1.0-p1.7 and P2.0-P2.7, which P1.0-P1.4 used to control DC motor, P1.0, P1.5 and P1.6 for receiving infrared signal module, P1.7 control rotary platform, P2.0-P2.3 for receiving the key signal, P2.4 and p2.5 for receiving and controlling ultrasonic module, p2.6 and p2.7 respectively receive mq-2 signal and control buzzer (see Fig.6-7).

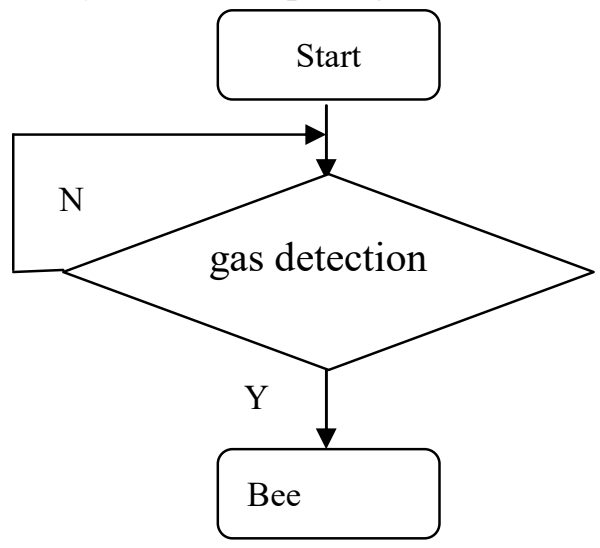

Fig. 6 Gas detection 


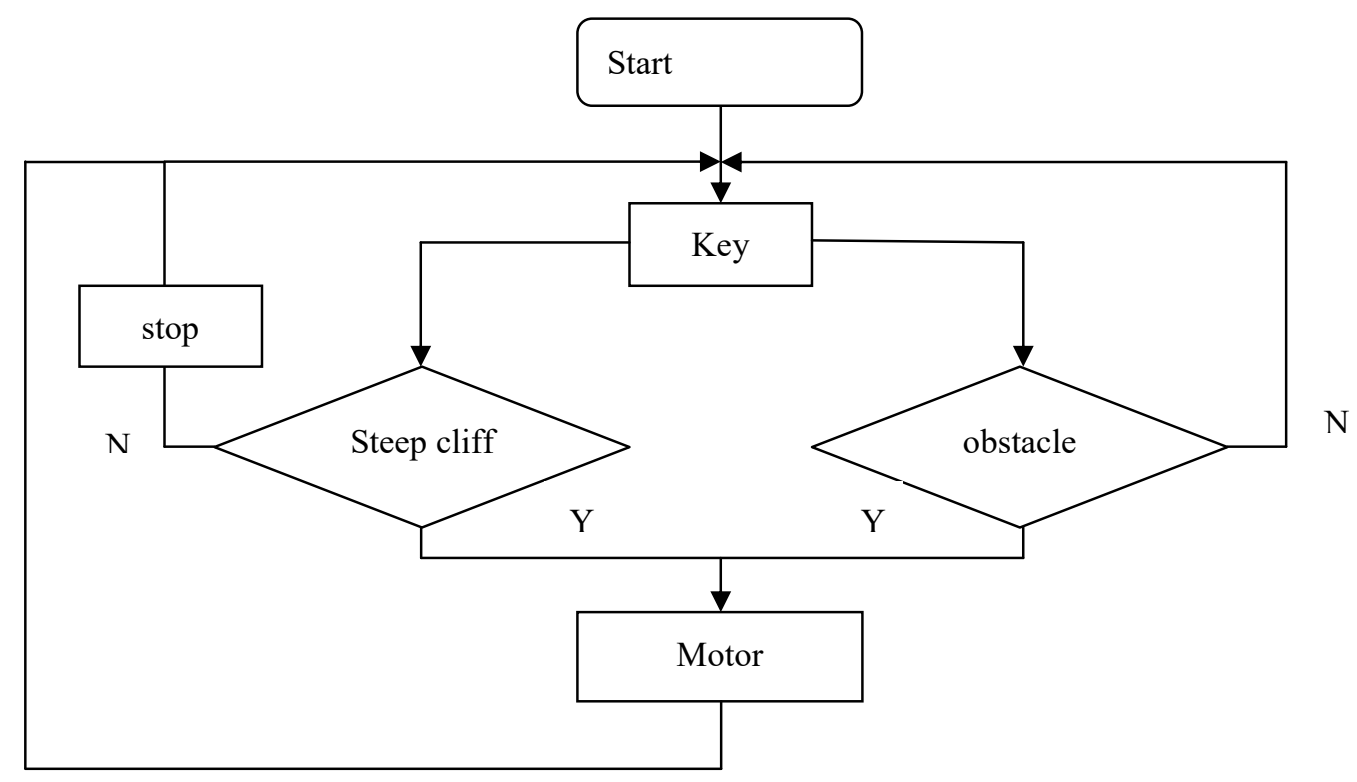

Fig.7 Procedure flow chart for obstacle avoidance

\section{Conclusions}

After testing, the intelligent wheelchair can realize the anti fall and the detection function of toxic gas, the basic realization of obstacle avoidance function, and can achieve a simple route planning. For complex line, due to the route algorithm, in which a large number of operations, the MSP430 microcontroller is bloated, with embedded module to optimize the design, it will be the future research contents.

\section{References}

[1] Jingchuan Wang,Weidong Chen,Wenlong Liao. An Improved Localization and Navigation Method for Intelligent Wheelchair In Narrow and Crowded Environments[J]. IFAC Proceedings Volumes,2013,4613:.

[2] Shuangshuang Lei. Fusing Visual Tracking and Navigation for Autonomous Control of An Intelligent Wheelchair[J]. IFAC Proceedings Volumes,2013,4620:.

[3] Mohammed Alshraideh,Basel A. Mahafzah,Saleh Al-Sharaeh,Ziad M. Hawamdeh. A robotic intelligent wheelchair system based on obstacle avoidance and navigation functions[J]. Journal of Experimental \& Theoretical Artificial Intelligence,2015,274:.

[4] Chaofeng Pan,Liao Chen,Long Chen,Haobin Jiang,Zhongxing Li,Shaohua Wang. Research on motor rotational speed measurement in regenerative braking system of electric vehicle[J]. Mechanical Systems and Signal Processing,2016,66-67:.

[5] Arshi Mohini. Microcontroller Based Orbital Motion Shaker[J]. Procedia Technology,2016,24:. 